\title{
Spinal epidural abscess from group A Streptococcus after varicella infection: a case report and review of the literature
}

\author{
G. Cossu • M. A. Farhane $\cdot$ R. T. Daniel $\bullet$ M. Messerer
}

Received: 16 May 2014 / Accepted: 26 June 2014 / Published online: 8 July 2014

(C) Springer-Verlag Berlin Heidelberg 2014

\begin{abstract}
Introduction Spinal epidural abscess (SEA) is a very rare condition in pediatric patients. Varicella zoster infection could be a predisposing factor, and SEA should be suspected in patients with signs of secondary bacterial infection and even mild neurological signs.

Clinical case We describe here a case of a 30-month-old girl with a history of remitting varicella infection, diagnosed for a lumbar epidural abscess and sacro-ileitis, secondary to group A Streptococcus (GAS).

Discussion This is the third case of SEA from GAS reported in the literature in a pediatric population with varicella infection. We discuss here the clinical presentation and the diagnostic challenges for SEA in childhood through a review of the literature.
\end{abstract}

Keywords Spinal epidural abscess · Varicella infection . Group A Streptococcus · Childhood

\section{Introduction}

Spinal epidural abscess (SEA) is a collection of pus or inflammatory tissue between the dura mater and the vertebral column. It is considered a medical emergency and it is a very rare condition with an incidence of $0.6 / 10,000$ admissions in the pediatric cohort [2]. SEA is mainly secondary to hematogenous spread with the primary entry point being skin or soft tissue infections and it is often associated with vertebral osteomyelitis and paraspinal abscess or systemic complications like endocarditis [4]. Predisposing conditions are immunosuppression,

G. Cossu $(\bowtie) \cdot$ M. A. Farhane $\cdot$ R. T. Daniel $\cdot$ M. Messerer

Departement de Neurosciences Cliniques, Service de

Neurochirurgie, Centre Hospitalier Universitaire Vaudois,

Université de Médecine et Biologie de Lausanne, Rue du Bugnon 46,

1011 Lausanne, Switzerland

e-mail: giulia.css@gmail.com diabetes, spinal surgery, or trauma [2]. The predominant etiological agent is Staphylococcus aureus [2, 11, 21] with an increasing prevalence of community-acquired methicillinresistant $S$. aureus (MRSA) infections in children without risk factors [9], followed by gram-negative bacteria.

Varicella infection may be a predisposing condition for SEA development in children: the varicella-zoster virus causes generally a self-limiting disease but severe bacterial complications have been reported in previously healthy children [6]. S. aureus and group A Streptococcus (GAS) are the major responsible: hematogenous spreading of GAS most frequently cause musculoskeletal infections, while epidural abscess were described more frequently in an adult population and are generally caused by $S$. aureus.

In our knowledge, we report here the third case described in the literature of SEA caused by GAS in a pediatric population with a positive history of varicella.

\section{Case report}

A previously healthy 30 -month-old girl, presented with a twoday history of high-grade fever, right leg pain, and refusal to walk. She had positive history for varicella infection during the last 10 days, followed by abdominal pain and rhinitis. On clinical examination, she was irritable; she showed a normal muscular strength despite her refusal to walk, with normal deep tendon reflexes and no neck stiffness. A throat culture done at admission grew GAS and blood tests showed an inflammatory syndrome and mild coagulopathy (WBC $11 \mathrm{~g} / 1$, platelets $458 \mathrm{~g} /$ 1, CRP $28 \mathrm{mg} / 1$, TP $65 \%$, aPTT $74 \mathrm{~s}$, fibrinogen $4.5 \mathrm{~g} / \mathrm{l}$ ). She was hospitalized for further investigations in a peripheral hospital with an antibiotic treatment (aqueous penicillin $\mathrm{G}$ intravenously). Her refusal to walk led to the realization of a lumbar MRI (with no gadolinium administration) showing an anterior epidural collection extending from L3 to S1, interpreted as a 


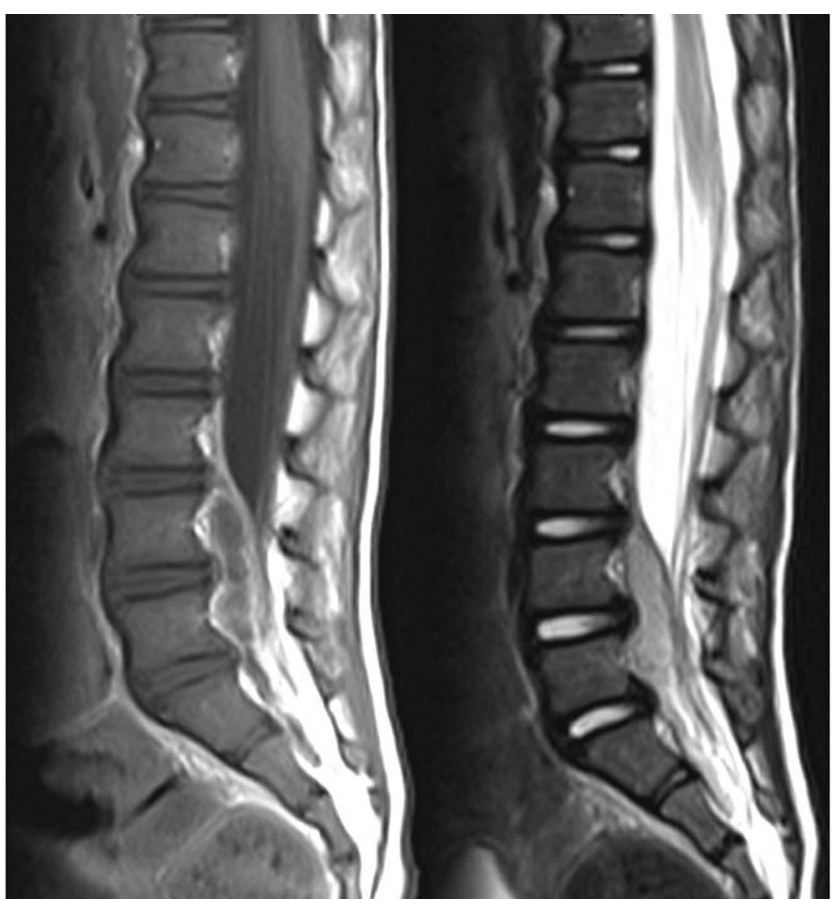

Fig. 1 Sagittal T1-weighted image showing a heterogeneous isointense collection in the anterior epidural space from the sacral column to the superior plate of L3 (left). Sagittal T2-weighted image showing the same isointense collection (right)

spontaneous epidural hematoma (Fig. 1) in the context of her coagulopathy, attributed to vitamin $\mathrm{K}$ deficiency.

Standard radiographies and pelvic echocardiography performed in the suspicion of osteitis or septic arthritis showed a non-specific peri-articular right sacro-ileal collection.

After her hospital admission of $24 \mathrm{~h}$, she was transferred to our department. A new pelvic and lumbosacral MRI with gadolinium administration revealed a right sacro-iliac osteomyelitis and a collection extending from the gluteal muscles to the presacral fat and through the right L5 and S1 foramina into the anterior epidural space till the superior plate of L3, with a strong contrast enhancement. The collection showed

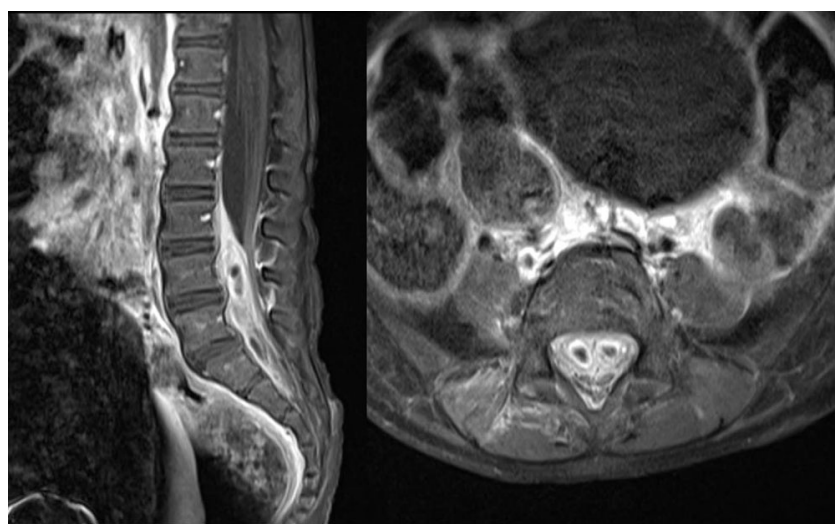

Fig. 2 Sagittal (left) and axial (right) T1-weighted images (with gadolinium) showing a collection with strong contrast enhancement, extending into the anterior epidural space till the superior plate of L3, with an important mass effect progression when compared to the previous MRI and it was finally interpreted as an epidural abscess (Fig. 2).

Because of a rapid neurological deterioration, the patient was taken to the emergency OR, where a bilateral laminoplasty of L3-S1 was performed with abscess drainage. Intraoperatively, a purulent material was isolated, surrounded by chronic inflammatory tissue. The initial cultures stayed negative but the gram stain was positive for a coccus and the PCR allowed identifying a GAS, sensitive to the current antibiotic treatment.

She was progressively mobilized after the intervention with a rapid recuperation of walking ability and bladder continence. At the end of the intravenous antibiotic course (6 weeks), she had a completely normal clinical status and an oral antibiotic treatment was started for another 6 weeks. The final lumbar MRI showed complete healing (Fig. 3).

\section{Discussion}

SEA is more common in the adult population (fifth to seventh decades) and in patients with underlying conditions like immunosuppression, diabetes, spinal surgery, or trauma $[2,15]$. These predisposing conditions occur more frequently in adults, and risk factors identified in the literature analysis for pediatric patients are sickle cell disease, Hirschsprung's disease, or chronic intestinal pseudo-obstruction [2]. Furthermore, varicella infection may represent an additional risk factor for SEA development.

SEA is more likely to develop in a larger epidural space rich in fat: abscesses are more common in posterior areas (above all in children) and in thoracolumbar segment. One third of pediatric patients had associated osteomyelitis [2]. In
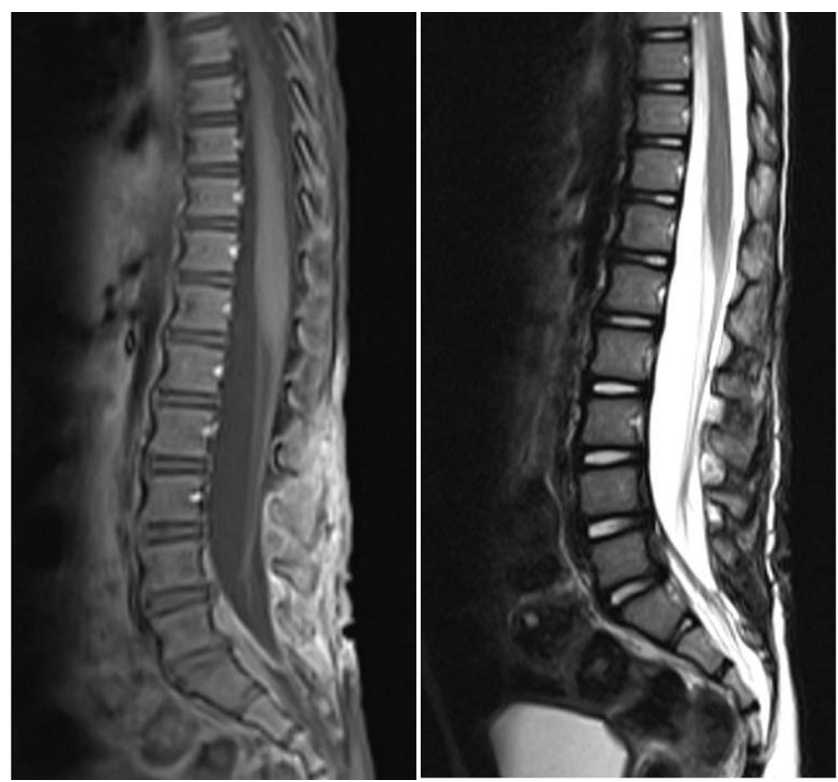

Fig. 3 Sagittal T1-weighted (left) and T2-weighted (right) lumbar MRI at 3-month follow-up, after the laminoplasty and the antibiotic therapy, showing a complete radiological healing 
$50 \%$ of cases, SEA is secondary to hematogenous dissemination from a remote focus (skin or soft tissue, urinary, or respiratory tract infection), and in 10-30\% of cases, it derives by contiguous spread (osteomyelitis or muscular abscess). In another $15 \%$ of cases, invasive procedures are implicated [5]. No source of predisposing illness can be identified in up to $30-40 \%$, suggesting silent bacteremia seeding the epidural space [20].

S. aureus is the predominant pathogen in SEA in children [2] with an increasing prevalence of MRSA during the last years [9], followed by gram-negative bacteria (especially Escherichia coli and Pseudomonas), coagulase negative Staphylococci (such as staphylococcus epidermidis), viridans group Streptococci, Enterococci, anaerobes, and Mycobacterium species [4].

Varicella-zoster virus (VZV) may further predispose SEA development. It is a ubiquitous, highly neurotropic, exclusively human $\alpha$-herpesvirus, and varicella infection is generally considered a mild and self-limiting disease commonly seen in primary care setting. It normally has a benign clinical course, and complications have been reported in 3-5\% of patients, with an estimated mortality rate at 1.4/100,000 [14]. Bacterial complications are the most prevalent (45-73\% of reported complications in varicella-zoster infection) [19] and they normally consist of skin and soft tissues involvement, with S. aureus and Streptococcus pyogenes being major responsible [1]. The pathogenesis of bacterial infection is thought to be due to skin barrier disruption and possibly transient virusinduced immunosuppression [1].

Our case is the third case described in the literature of pediatric SEA from GAS in the context of varicella infection. In our review of the literature, two cases of SEA from GAS were reported in two pediatric patients [15] and one case of SEA caused by $S$. aureus was reported in a 2-year-old girl with a positive history of varicella (Table 1) [7]. Two other cases of SEA from GAS were described in two pediatric patients with no varicella infection $[13,16]$.

Our patient presented with recurrent high fever, she was very irritable, and she refused to walk. The first lumbar MRI without contrast administration showed an anterior epidural collection extending from the sacrum to the superior plate of L3. The signal was isointense in both T1- and T2-weighted images, and the collection was dismissed for a spontaneous hematoma in the context of her coagulopathy (Fig. 1). A second pelvic-lumbar MRI with gadolinium administration was performed because of the strong suspicion of an infectious focus and it showed a right sacro-iliac osteomyelitis and a lumbar abscess in the anterior epidural space extending by contiguity from the arthritis focus. Urgent surgical decompression was necessary for a rapid neurological deterioration and GAS was isolated from intraoperative material.

The clinical presentation in SEA can be misleading. Heusner et al. [10] first described the classical symptoms

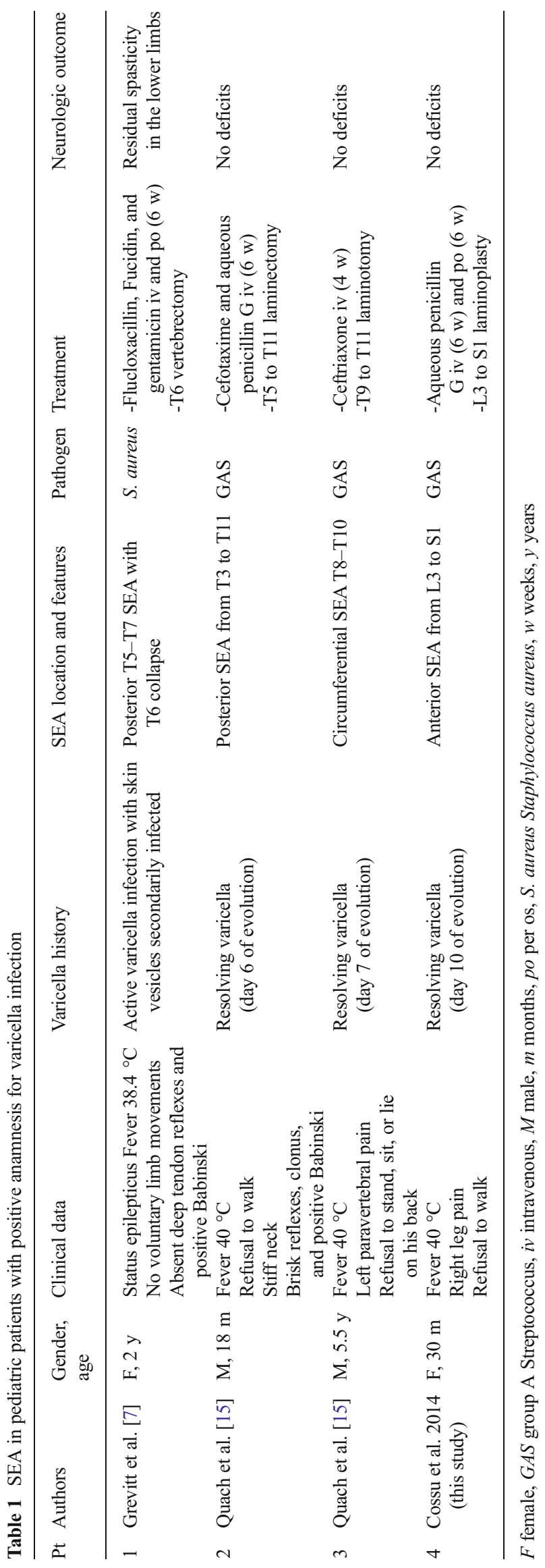


associated to epidural abscess: fever and back pain, followed by neurological manifestations. Unfortunately, only a minority of cases presents with the classical triad [17], and in children, the diagnosis is more challenging. A high index of suspicion is necessary in pediatric patients with a positive history for varicella infection (either acute or remitting), clinical signs of secondary bacterial infection, high-grade fever, and even mild neurological symptoms. With these conditions, it is imperative to be attentive to any sign of neurological deterioration and it is advisable to investigate the presence of epidural abscess with an adequate imaging.

The differential diagnosis includes other infectious process like myelitis or meningitis and parainfectious process. Rubin et al. [18] performed lumbar punctions (LP) in a cohort of patients with SEA and they found clear infectious signs only in $52 \%$ of cases. No specific results were shown in $38 \%$ of cases and CSF was normal in $10 \%$ of cases. Other authors [3, 15] performed routinely LP for SEA patients. In our opinion, LP is an unnecessary procedure, which stays non informative in half of cases and which may increase the risk of bacterial dissemination in the central nervous system. The therapeutic decisions are not modified by the results of CSF analysis, and according to our point of view, it should not be routinely performed before a correct radiological assessment.

The main determinant of final outcome is the preoperative neurologic status [2, 18], and standard approach lies in surgical decompression and drainage in combination with several weeks of intravenous (4-6 weeks) [15] and oral antibiotics (4-6 weeks). Some studies [12, 21] affirm that a conservative treatment may be the best option for selected candidates with no neurological symptoms or with complete deficits lasting more than $72 \mathrm{~h}$, with extensive abscess or with high surgical risks $[8,12,13,17]$. However, with conservative treatment, a risk of deterioration even in patients without neurologic symptoms was reported $[2,21]$. Pediatric patients seem to have a better prognosis even if they present with a more extended disease [2].

In our opinion, SEA is a potentially fatal illness necessitating prompt diagnosis, and the best therapeutic option in the pediatric setting is a combination of surgery and long-term antibiotic therapy. In particular, the surgical decompression is advisable even when the neurological deficit is present for more than $72 \mathrm{~h}$.

\section{Conclusion}

SEA is a very rare condition in children, and varicella infection may be a predisposing factor. Our report is the third case described in the literature of pediatric SEA from GAS infection in the context of varicella. Childhood SEA is a challenging diagnosis, and according to our findings, spinal MRI should be performed in every patient having varicella infection with bacterial complications or coexisting risk factors and neurological symptoms. The treatment should combine an aggressive surgical approach with a long-course antibiotic therapy.

Conflict of interest The authors declare that they have no conflict of interest.

\section{References}

1. Aebi C, Ahmed A, Ramilo O (1996) Bacterial complications of primary varicella in children. Clin Infect Dis 23:698-705

2. Auletta JJ, John CC (2001) Spinal epidural abscesses in children: a 15-year experience and review of the literature. Clin Infect Dis 32:916

3. Danner RL, Hartman BJ (1987) Update on spinal epidural abscess: 35 cases and review of the literature. Rev Infect Dis 9:265-274

4. Darouiche RO (2006) Spinal epidural abscess. N Engl J Med 355: 2012-2020

5. Darouiche RO, Goetz L, Kaldis T, Cerra-Stewart C, AlSharif A, Priebe M (2006) Impact of StatLock securing device on symptomatic catheter-related urinary tract infection: a prospective, randomized, multicenter clinical trial. Am J Infect Control 34: $555-560$

6. Fleisher G, Henry W, McSorley M, Arbeter A, Plotkin S (1981) Lifethreatening complications of varicella. Am J Dis Child 135:896-899

7. Grevitt MP, Mehdian SH (1998) Epidural abscess in an infant. Eur Spine J 7:413-415

8. Hawkins M, Bolton M (2013) Pediatric spinal epidural abscess: a 9year institutional review and review of the literature. Pediatrics 132: e1680-e1685

9. Herold BC, Immergluck LC, Maranan MC, Lauderdale DS, Gaskin RE, Boyle-Vavra S, Leitch CD, Daum RS (1998) Communityacquired methicillin-resistant Staphylococcus aureus in children with no identified predisposing risk. JAMA 279:593-598

10. Heusner AP (1948) Nontuberculous spinal epidural infections. N Engl J Med 239:845-854

11. Karikari IO, Powers CJ, Reynolds RM, Mehta AI, Isaacs RE (2009) Management of a spontaneous spinal epidural abscess: a singlecenter 10-year experience. Neurosurgery 65:919-923, discussion 923-914

12. Leys D, Lesoin F, Viaud C, Pasquier F, Rousseaux M, Jomin M, Petit H (1985) Decreased morbidity from acute bacterial spinal epidural abscesses using computed tomography and nonsurgical treatment in selected patients. Ann Neurol 17:350-355

13. Nussbaum ES, Rigamonti D, Standiford H, Numaguchi Y, Wolf AL, Robinson WL (1992) Spinal epidural abscess: a report of 40 cases and review. Surg Neurol 38:225-231

14. Preblud SR (1986) Varicella: complications and costs. Pediatrics 78: 728-735

15. Quach C, Tapiero B, Noya F (2002) Group A streptococcus spinal epidural abscess during varicella. Pediatrics 109:E14

16. Renoux MC, Guyon G, Rodiere M (2006) Paravertebral streptococcal myositis complicated by an epidural abscess in a 5-year-old girl. Arch Pediatr 13:273-275 
17. Rigamonti D, Liem L, Sampath P, Knoller N, Namaguchi Y, Schreibman DL, Sloan MA, Wolf A, Zeidman S (1999) Spinal epidural abscess: contemporary trends in etiology, evaluation, and management. Surg Neurol 52:189-196, discussion 197

18. Rubin G, Michowiz SD, Ashkenasi A, Tadmor R, Rappaport ZH (1993) Spinal epidural abscess in the pediatric age group: case report and review of literature. Pediatr Infect Dis J 12:10071011
19. Saddier P, Floret D, Guess HA, Durr F, Peyrieux JC, Weber DJ, Plotkin SA (1998) Cost of varicella in France: a study in day care centers. J Infect Dis 178(Suppl 1):S58-S63

20. Sendi P, Bregenzer T, Zimmerli W (2008) Spinal epidural abscess in clinical practice. QJM 101:1-12

21. Wheeler D, Keiser P, Rigamonti D, Keay S (1992) Medical management of spinal epidural abscesses: case report and review. Clin Infect Dis 15:22-27 